\title{
On the dispersion law of low-frequency electron whistler waves in a multi-ion plasma
}

\author{
B. V. Lundin ${ }^{1}$ and C. Krafft ${ }^{2}$ \\ ${ }^{1}$ Institute of Terrestrial Magnetism, Ionosphere and Radiowave Propagation, Russian Academy of Sciences, Troitsk, Moscow \\ Region, 142190, Russia \\ ${ }^{2}$ Laboratoire de Physique et Technologie des Plasmas, Ecole Polytechnique, 91128 Palaiseau Cedex, France
}

Received: 5 October 2007 - Accepted: 20 February 2008 - Published: 11 June 2008

\begin{abstract}
A new and simple dispersion law for extra-lowfrequency electron whistler waves in a multi-ion plasma is derived. It is valid in a plasma with finite ratio $\omega_{c} / \omega_{p e}$ of electron gyro-to-plasma frequency and is suitable for wave frequencies much less than $\omega_{p e}$ but well above the gyrofrequencies of most heavy ions. The resultant contribution of the ions to the dispersion law is expressed by means of the lower hybrid resonance frequency, the highest ion cutoff frequency and the relative content of the lightest ion. In a frequency domain well above the ions' gyrofrequencies, this new dispersion law merges with the "modified electron whistler dispersion law" determined in previous works by the authors. It is shown that it fits well to the total cold plasma electron whistler dispersion law, for different orientations of the wave vectors and different ion constituents, including negative ions or negatively charged dust grains.
\end{abstract}

Keywords. Ionosphere (Wave propagation) - Radio Science (Waves in plasma) - Space Plasma Physics (Waves and instabilities)

\section{Introduction}

One of the typical problems inherent to the application of the Geometrical Optics (GO) or the ray approximation approach consists in the fact that the expression for the local dispersion equation of plane waves of the magnetoactive plasma modes under consideration should be known; actually, one should provide the data about the plasma constituents contributing to the local dielectric permittivity tensor along the rays' paths. This problem is very real in the near-Earth's plasma surroundings, where the waves typically used in a global passive or active sounding belong to the electron whistler frequency range. Conventionally,

Correspondence to: C. Krafft

(catherine.krafft@1ptp.polytechnique.fr) they cover the so-called very-low-frequency (VLF, $3 \mathrm{kHz}-$ $30 \mathrm{kHz}$ ) and extremely-low-frequency (ELF, $300 \mathrm{~Hz}-3 \mathrm{kHz}$ ) domains; however, in recent active space investigations, their frequency range was extended up to $3 \mathrm{MHz}$ (Helliwell, 1965, 1993; Kimura, 1985; Reinish, 2000). In an inhomogeneous magnetospheric plasma, the sources of whistler emissions can be located far from the region where the wave emissions effectively reveal their properties and are registered by remote receivers. Then the GO approach is conventionally used to solve the problem of the spectral characteristics' evolution of the emissions during their distant propagation from their sources; it was also used effectively in the recent active radio sounding projects similar to that one realized on board the IMAGE satellite (Burch, 2000; Reinish, 2000; Sonwalkar et al., 2004; Carpenter, 2004). Moreover, the GO technique was also applied to fit the parameters of space density model distributions using the Direction Finding Data of groundbased transmitter signals registered on board the "Ikebono" satellite (Sawada et al., 1993).

An adjacent problem typical of the ray approximation approach consists of simplifying a general plasma dispersion law in order to reduce, if possible, the amount of the physical parameters governing explicitly the wave propagation of a selected plasma mode. To solve this problem, instead of the unknown space distributions of the different plasma constituents, one can use several inherent plasma frequencies which are often visible as peculiarities of the electromagnetic spectra registered on board space vehicles (Benson et al., 2003). In general, a relevant simplification of the total cold plasma dispersion law is a very complicated analytical task, as the magnetospheric plasma parameters can vary by two orders of magnitude along a single ray path. For example, the frequency of a wave belonging to the lower hybrid resonance (LHR) domain, around $10 \mathrm{kHz}$ (resp. to the proton gyrofrequency domain, around $500 \mathrm{~Hz}$ ) near the Earth, can become comparable with the electron gyrofrequency $\omega_{c}$ (resp. with the LHR frequency $\omega_{l h r}$ ) at the equatorial top of the

Published by Copernicus Publications on behalf of the European Geosciences Union. 
geomagnetic field line at the altitude of three Earth's radii. Fortunately, for electron whistler waves of sufficiently high frequencies the problem was solved by Lundin and Krafft (2001, 2002), who showed that the resultant contribution of all the ions can be reduced to the value of the LHR frequency only, if the whistler frequency essentially exceeds the local gyrofrequency of the lightest ion. At the same time, the limiting condition of overdense plasma (inherent to the conventional whistler dispersion law, Shafranov, 1967) was overcome and the modified electron whistler dispersion law which was obtained is applicable for a finite ratio $\omega_{p e} / \omega_{c}$, where $\omega_{p e}$ is the electron plasma frequency. This condition is indispensable, particularly for the near-Earth plasma at altitudes where the transition occurs from the lightest ions' to the more heavy ions' prevalence, i.e. typically at altitudes of few thousands kilometers above the Earth (Sonwalkar et al., 2004).

Thus, in a very wide frequency domain localized well above the gyrofrequency of the lightest ion, the dispersion of the electron whistler waves is governed by the frequencies $\omega_{l h r}, \omega_{c}$ and $\omega_{p e}$. For the extra-low-frequency domain, which is closer to the lightest ion gyrofrequency near the Earth, a reliable and simple dispersion relation has not been found up to now. However, we will show that if additional data are available, namely the highest ion cutoff frequency and the relative content of the lightest ions, then the problem can be solved with a relevant accuracy (see also Smith and Brice, 1964; Brice and Smith, 1965; Gurnett and Burns, 1968; Muzzio, 1968; Muzzio and Angerami, 1972; Santolic and Parrot, 1998, and references therein).

It is well known that the peculiarities of the wave dispersion laws in the low frequency range are essentially different for a single ion plasma or a multi-ion species plasma (Hines, 1957; Buchsbaum, 1960; Yakimenko, 1962; Gintzburg, 1963; Smith and Brice, 1964; Gurnett and Burns, 1968; Das and Uberoi, 1972; Mamun et al., 2004, 2005, and references therein). In the latter case, the main feature for plane waves is the appearance of cutoff frequencies in the domains of the ions' gyrofrequencies and of resonance frequencies, depending on their polarization sense. The possible influence of plasma rotation, accompanied by additional force-terms in the equations of plasma motion (Kashyapi et al., 1993, and references therein), was also discussed in the application to the wave dispersion features. In a paper of Ganguli and Rudakov (2004) the physical nature of the cutoff phenomena in the ions' gyrofrequencies' domains was clarified, for the first time, in a plasma with heavy negatively charged particles; it corresponds to the first order mobile ion fluid rotation with a cutoff frequency in a plasma where the electron density is not equal to the density of the mobile positive ions (in the presence of immobile negative dust grains; see also Pandey and Vranjes, 2006). The peculiarities of the nonlinear processes occurring in the low frequency domain of a dense multi-ion plasma have been considered in many papers (e.g. see Faria et al., 1998; Irie and Ohsawa,
2003; Hertzberg et al., 2004, and references therein), mainly in application to the different types of Alfvén waves with frequencies lower than the proton gyrofrequency (for higher frequency domains, e.g. see Yu et al., 1974; Stenzel, 1999, and references therein). A general dispersion law in a framework of Hall-magnetohydrodynamic plasmas with pressure terms was presented by Shukla et al., 2005, where the contributions of electrons, one type of ion and immobile charged dust particulates have been taken into account. However, the omitted displacement current components orthogonal to the background magnetic field restrict its application for the electron whistler waves only to the case of overdense plasmas. To the best of our knowledge, an expression for the electron whistler dispersion law which is reliable in the ions' cutoff frequencies' domain (and substantially above it), particularly in the presence of some significative amount of negative ions, has not been reported yet in the literature.

In this paper we derive an electron whistler dispersion law which is suitable for the lower frequency range till the highest ion cutoff frequency. It is demonstrated that it fits well with the total cold plasma dispersion law in an extra wide frequency domain extending over three orders of frequency values, so that in the high frequency domain the new dispersion law merges with the modified electron whistler dispersion law, which was determined recently by the authors (Lundin and Krafft, 2001, 2002) and which is applicable till the domain of the electron gyrofrequency. It is shown that, in a plasma with sufficiently separated values of the ions' gyrofrequencies, the new dispersion equation for the whistler range can be reduced to a biquadratic equation in frequency. In this case it is sufficient to provide two additional external parameters only, namely the values of the highest ion cutoff frequency and of the relative content of the lightest ion, hydrogen, for example. The possibility to increase significantly the value of the cutoff frequency above the lightest ion gyrofrequency in the presence of negative ions (or dust grains) is discussed in an application to the problem of electron whistler wave propagation and reflection in the upper ionosphere.

\section{Electron whistler dispersion law in the lightest ion's gyrofrequency domain}

Let us derive a reliable expression for the electron whistler wave dispersion law which is suitable for the frequency domain around the lightest ion's gyrofrequency - i.e. the gyrofrequency $\omega_{H}$ of hydrogen $\mathrm{H}^{+}$, for example. Actually, at higher frequencies $\omega \gg \omega_{H}$, such a dispersion law should be close to the modified electron whistler dispersion law derived for a plasma with finite $\omega_{p e} / \omega_{c}$ (Lundin and Krafft, 2001, 2002), which, in turn, is applicable from the near LHR frequency range till the high frequency domain, where one can disregard the contribution of the ions. The best way to derive a new dispersion law reliable for extra-low-frequency 
electron whistler waves in a multi-ion plasma is to use a cold plasma dispersion law in the form where the term responsible for the ions' cutoff frequencies visibly appears. Then, after suitable simplifications, the resultant extra-low-frequency whistler dispersion law $\omega(\boldsymbol{k})$ can be obtained as the largest root of a biquadratic equation.

The usual cold plasma dispersion equation (without any simplifications) can be written in the following form

$$
\begin{aligned}
& K\left(\frac{\digamma}{(\varepsilon-\eta)}\left(K+\sin ^{2} \theta\right)+\cos ^{2} \theta_{R}\left(1+\cos ^{2} \theta\right)\right) \\
& \quad+\cos ^{2} \theta_{R}-\cos ^{2} \theta=0,
\end{aligned}
$$

with

$$
\begin{aligned}
\frac{\digamma}{(\varepsilon-\eta)} & =\cos ^{2} \theta_{R}+\frac{\chi}{\eta}=\frac{\varepsilon^{2}-g^{2}}{\eta(\varepsilon-\eta),} \\
K & =-\eta / N^{2}, \quad \cos ^{2} \theta_{R}=\frac{\varepsilon}{\varepsilon-\eta},
\end{aligned}
$$

where $N=c k / \omega$ is the refractive index, $c$ is the light velocity, $k$ is the wave vector modulus, $\omega$ is the frequency and $\theta$ is the angle between the wave vector $\boldsymbol{k}$ and the constant background magnetic field $\boldsymbol{B}_{0}, \cos \theta=\boldsymbol{k} \cdot \mathbf{B}_{0} / k B_{0}$. The so-called cutoff points of the refractive index $(N \rightarrow 0$ or $K \rightarrow \infty)$ can be reached only at the cutoff frequencies where $\digamma(\omega)=0$ (Stix, 1992). The expression for $\cos ^{2} \theta_{R}$ actually corresponds to the formal definition of the resonance angle $\theta_{R}$, i.e. $\varepsilon \sin ^{2} \theta_{R}+\eta \cos ^{2} \theta_{R}=0$ (where, for $\cos ^{2} \theta_{R}<0$ or $\cos ^{2} \theta_{R}>1$, the resonance cone does not exist for plane waves); the parameter $\chi$ (useful in the high frequency domain, where it tends to unity) is

$\chi=\varepsilon-\frac{g^{2}}{\varepsilon-\eta}$.

The components $\varepsilon, g$ and $\eta$ of the dielectric permittivity tensor in a cold plasma (Shafranov, 1967) are defined by

$$
\begin{aligned}
& \varepsilon=1-\sum_{j} \frac{\omega_{p j}^{2}}{\omega^{2}-\omega_{c j}^{2}}, \\
& g=-\sum_{j} \frac{\omega_{p j}^{2} \omega_{c j}}{\omega\left(\omega^{2}-\omega_{c j}^{2}\right)}, \\
& \eta=1-\sum_{j} \frac{\omega_{p j}^{2}}{\omega^{2}},
\end{aligned}
$$

where $\omega_{p j}$ and $\omega_{c j}$ are the plasma and the gyro-frequencies of the species $j$, respectively

$\omega_{p j}^{2}=Z_{j}^{2} \frac{4 \pi n_{j} e^{2}}{M_{j}}, \quad \omega_{j}=\frac{Z_{j} e B_{0}}{M_{j} c}, \quad Z_{j}, e>0$,

$\omega_{p e}^{2}=\frac{4 \pi n_{e} e^{2}}{m}, \quad \omega_{c}=\frac{e B_{0}}{m c}, \quad \omega_{p H}^{2}=\frac{4 \pi n_{H} e^{2}}{M}$.

For electrons one uses the index " $e$ " and the notations $\omega_{c} \equiv-\omega_{c e}>0$; for negative ions (index " $\beta$ "), we note $\omega_{c \beta} \equiv-\omega_{\beta}<0$, and for positive ions (index “ $\alpha$ "), $\omega_{c \alpha} \equiv \omega_{\alpha}>0$. Thus $\omega_{\alpha}$ and $\omega_{\beta}$ are both positive for any sign of the ions' charge, whose value in electron charge units is $Z_{j}>0 ; n_{j}$ and $M_{j}$ are the density and the mass of the ion species $j$; for electrons, we note $m \equiv M_{e}$, and for protons, $M \equiv M_{H}$.

For the case of a rather dense plasma, where the displacement current component parallel to the background magnetic field can be neglected in the frequency band under consideration, we have

$$
\begin{aligned}
-\frac{\eta}{N^{2}} & =K=-\frac{\omega^{2}}{k^{2} c^{2}}+\frac{\omega_{p i}^{2}}{k^{2} c^{2}}+\frac{\omega_{p e}^{2}}{k^{2} c^{2}} \simeq \frac{\omega_{p}^{2}}{k^{2} c^{2}} \frac{n_{e}}{n} \equiv \varkappa, \\
\omega_{p i}^{2} & \equiv \sum_{j} \omega_{p j}^{2},
\end{aligned}
$$

where $\omega_{p}$ is the value of the electron plasma frequency calculated for an electron density $n_{e}$ equal to the total density $n$ of the positive charges, which is greater than $n_{e}$ in the presence of negative ions (or negative dust grains). In this paper, we do not restrict ourselves to the case of an overdense plasma and thus the ratio $\omega_{c}^{2} / \omega_{p}^{2}$ is of the order of unity and should not be considered as vanishingly small. The charge neutrality condition is

$n=\sum_{\alpha} Z_{\alpha} n_{\alpha}=n_{e}+\sum_{\beta} Z_{\beta} n_{\beta}$.

Actually an additional rather soft limitation, $\omega_{p e}^{2} \gg \omega_{p i}^{2}$, i.e. $n_{e} / n \gg(m / M)(Z / M)_{\mathrm{eff}}$, is applied in Eq. (6), with

$$
(Z / M)_{\mathrm{eff}} \equiv \sum_{j} \frac{Z_{j} n_{j}}{n} \frac{Z_{j} M}{M_{j}}, \quad \sum_{j} \frac{Z_{j} n_{j}}{2 n-n_{e}}=1,
$$

where the summation over $j$ includes both positive and negative ions (compare with the Appendix B); without negative ions one has $n_{e}=n$.

The function $\digamma(\omega)$ is a finite constant in the frequency domain $\omega_{j} \ll \omega \ll \omega_{c}$, namely

$$
\begin{gathered}
\digamma(\omega)=\frac{\varepsilon^{2}-g^{2}}{\eta} \simeq \frac{\omega_{p e}^{2}}{\omega_{c}^{2}}\left(1-\chi \frac{\omega^{2}}{\omega_{p e}^{2}}\left(2+\chi \frac{\omega_{c}^{2}}{\omega_{p e}^{2}}\right)\right) \\
\left(1-\chi \frac{\omega^{2}}{\omega_{p e}^{2}}\right)^{-1} \simeq \frac{\omega_{p e}^{2}}{\omega_{c}^{2}} \equiv \mu^{-1},
\end{gathered}
$$

and a plasma with small $\mu$ is named as overdense. In the same frequency range, one can find that

$$
\begin{aligned}
\varepsilon & \simeq 1+\frac{\omega_{p e}^{2}}{\omega_{c}^{2}}-\frac{\omega_{p i}^{2}}{\omega^{2}}, \quad \varepsilon-\eta \simeq \frac{\omega_{p e}^{2}}{\omega^{2}} \gg \varepsilon, \\
\chi \frac{\omega^{2}}{\omega_{p e}^{2}} & \simeq \frac{\omega^{2}}{\omega_{p e}^{2}}\left(1-\frac{\omega_{p i}^{2}}{\omega^{2}}\right) \ll 1, \quad \eta \simeq-\frac{\omega_{p e}^{2}}{\omega^{2}}, \\
\varepsilon \pm g & \simeq 1-\frac{\omega_{p i}^{2}}{\omega^{2}}-\frac{\omega_{p e}^{2}}{\omega\left(\omega \pm \omega_{c}\right)},
\end{aligned}
$$


where the summation over $j$ in Eq. (6) for $\omega_{p i}^{2}$ includes positive and negative ions as well as dust particles of any charge. Under the conditions (9-10) the electron whistler dispersion law can be found in the form of the modified dispersion law obtained by Lundin and Krafft (2002) (see also Eq. (25) below), where the resultant contribution of all the ions reduces to $\omega_{p i}$ or $\omega_{l h r} ; \omega_{l h r}$ (27) is not the exact solution of $\varepsilon\left(\omega_{l h r}\right)=0$, but a simplified expression calculated using the approximation $\omega_{H} \ll \omega \ll \omega_{c}$, as done for the first time by Smith and Brice (1964).

For calculating $\digamma(\omega)=(\varepsilon-g)(\varepsilon+g) / \eta$ in the extra-lowfrequency domain close to the ions' gyrofrequencies, i.e. $\omega \simeq \omega_{j}$, we use the approximations

$$
\begin{gathered}
\varepsilon \pm g=1 \mp \frac{n}{n_{e}} \frac{\omega_{p e}^{2}}{\omega \omega_{c}} \\
\left(-v_{e} \frac{\omega}{\omega \pm \omega_{c}}-\sum_{\beta} v_{\beta} \frac{\omega}{\omega \pm \omega_{\beta}}+\sum_{\alpha} v_{\alpha} \frac{\omega}{\omega \mp \omega_{\alpha}}\right) \\
\simeq 1+\frac{\omega_{p e}^{2}}{\omega_{c}^{2}} \mp \frac{\omega_{p e}^{2}}{\omega \omega_{c}}\left(1+\sum_{\beta} \frac{v_{\beta} / v_{e}}{1 \pm \omega / \omega_{\beta}}-\sum_{\alpha} \frac{v_{\alpha} / v_{e}}{1 \mp \omega / \omega_{\alpha}}\right),
\end{gathered}
$$

where $v_{j}=Z_{j} n_{j} / n>0$. Then, in the extra-low-frequency domain where $\omega \simeq \omega_{H} \ll \omega_{c}$, and for $\omega_{p}^{2} / \omega_{c}^{2} \sim 1$ (for plasmas which are at least moderately dense, $\omega_{p e}^{2} / \omega \omega_{c} \gg 1+\omega_{p e}^{2} / \omega_{c}^{2}$, see Smith and Brice, 1964), one can estimate the cutoff frequencies in the zero order approximation from the equations (see also the Appendix B)

$$
\begin{aligned}
& \digamma_{m}(\omega)=\sum_{\alpha} v_{\alpha} \frac{\omega}{\omega+\omega_{\alpha}}-\sum_{\beta} v_{\beta} \frac{\omega}{\omega-\omega_{\beta}}-v_{e} \frac{\omega}{\omega-\omega_{c}}=0 \\
& \digamma_{p}(\omega)=\sum_{\alpha} v_{\alpha} \frac{\omega}{\omega-\omega_{\alpha}}-\sum_{\beta} v_{\beta} \frac{\omega}{\omega+\omega_{\beta}}-v_{e} \frac{\omega}{\omega+\omega_{c}}=0 .
\end{aligned}
$$

However, to obtain more accurate numerical estimates for a plasma with small relative electron density, $n_{e} / n \ll 1$, so that $\omega_{p e}^{2} / \omega_{c}^{2} \ll 1$, when the highest ion cutoff frequency can tend to $\omega_{l h r}$ (as below in Fig. 3), one needs to use the equation $\digamma(\omega)=0$ in a more complete form

$\digamma(\omega)=\frac{n}{n_{e}} \frac{\omega_{p}^{2}}{\omega_{c}^{2}} \digamma_{m} \digamma_{p}+\frac{n}{n_{e}} \frac{\omega}{\omega_{c}}\left(\digamma_{p}-\digamma_{m}\right)-\frac{n}{n_{e}} \frac{\omega^{2}}{\omega_{p}^{2}}=0$.

But actually, if the relative electron density is not very small, i.e. for $n_{e} / n \gtrsim 0.05$, the last term of Eq. (15) can be omitted (see the Appendix B). The condition $n_{e} / n \ll 1$ (and $\left.\omega_{p e}^{2} / \omega_{c}^{2} \ll 1\right)$ can be typical of a plasma enriched by a noticeable amount of heavy negatively charged particles, due to the presence of electronegative gas ions or dust grains that are strongly adherent to the electrons (Smith, 1965; An et al., 1993; Kashyapi et al., 1993, for references). The presence of such plasma regions can be detected owing to the spectral features of the registered emissions, even if this local plasma state can be considered only as transient.

To illustrate our general approach to derive a dispersion law, let us simplify Eq. (15) for a plasma with two different positive ion species only (hydrogen $\mathrm{H}^{+}$and oxygen $\mathrm{O}^{+}$, for example, $\left.\omega_{1}=\omega_{H}, \omega_{2}=\omega_{O}, n_{e}=n=n_{H}+n_{O}\right)$. Then, from Eq. (14)

$$
\begin{aligned}
& \digamma_{p}(\omega) \propto \omega\left(\omega_{c}+v_{O} \omega_{O}+v_{H} \omega_{H}\right) \\
& -v_{H} \omega_{c} \omega_{O}-v_{O} \omega_{c} \omega_{H}-\omega_{O} \omega_{H}=0,
\end{aligned}
$$

we get the ion cutoff frequency $\omega_{\text {cut }}$

$\omega_{\mathrm{cut}} \simeq \frac{n_{O}}{n} \omega_{H}+\frac{n_{H}}{n} \omega_{O}$.

One has $\omega_{O}<\omega_{\text {cut }}<\omega_{H}$ and, as is well known, in the regions where the hydrogen ions are prevalent $\left(n_{H} \gg n_{O}\right), \omega_{\text {cut }}$ is close to the gyrofrequency of the more heavy ion-oxygen here (and vice versa). Then, Eq. (15) leads to

$$
\begin{aligned}
\digamma(\omega) & \simeq \frac{n}{n_{e}} \frac{\omega_{p}^{2}}{\omega_{c}^{2}} \digamma_{m} \digamma_{p}=\frac{\omega_{p e}^{2}}{\omega_{c}^{2}} \frac{\omega^{2}\left(\omega^{2}-\omega_{\mathrm{cut}}^{2}\right)}{\left(\omega^{2}-\omega_{H}^{2}\right)\left(\omega^{2}-\omega_{O}^{2}\right)} \\
& \simeq \frac{\omega_{p e}^{2}}{\omega_{c}^{2}} \frac{\left(\omega^{2}-\omega_{\mathrm{cut}}^{2}\right)}{\left(\omega^{2}-\omega_{H}^{2}\right)}, \\
\digamma(\omega) & =\frac{\omega_{p e}^{2}}{\omega_{c}^{2}}\left(1+\frac{\omega_{H}^{2}-\omega_{\mathrm{cut}}^{2}}{\omega^{2}-\omega_{H}^{2}}\right), \\
\omega^{2} & \simeq \omega_{H}^{2} \gg \omega_{O}^{2}, \quad \omega_{p e}^{2}=\omega_{p}^{2},
\end{aligned}
$$

where only one resonant term at $\omega_{H}$ is conserved finally for the frequency domain $\omega^{2} \gg \omega_{O}^{2}$, i.e. well above the gyrofrequency of the more heavy charged plasma particles.

The more general case when the plasma contains two types of positive ions and one type of heavy negatively charged particles is considered in Appendix B; however, the most simple resultant expression for $\digamma(\omega)$ can be reduced to Eq. (19), if the highest cutoff frequency $\omega_{\text {cut }}$ sufficiently exceeds the other ions' cutoff and resonant frequencies. The expression for the analytical estimate of $\omega_{\text {cut }}$ requires that the ions' species content data should be available; if those are not, then the value of the highest ion cutoff frequency should be taken from the registered spectra as the value of the lower cutoff frequency for the electron whistler waves.

\section{Extra-low-frequency dispersion law}

Actually, the parameter $K$ in Eq. (1) is very close to $\varkappa=\omega_{p e}^{2} / k^{2} c^{2}$ (with an error not larger than $\left.\omega_{p i}^{2} / \omega_{p e}^{2} \sim\left(n / n_{e}\right)(m / M)\right)$, so that one can write the dispersion Eq. (1) in the form

$\varkappa\left[\digamma\left(\varkappa+\sin ^{2} \theta\right)+\varepsilon\left(1+\cos ^{2} \theta\right)\right]+\varepsilon-(\varepsilon-\eta) \cos ^{2} \theta=0$.

A crucial simplification of Eq. (20) can be carried out only if the single resonant term proportional to $1 /\left(\omega^{2}-\omega_{j}^{2}\right)$ (where 
$\omega_{j}$ is the gyrofrequency of the lightest ion, i.e. $\omega_{j}=\omega_{H}$ here) is conserved finally in $\varepsilon(\omega)$ and $\digamma(\omega)$

$$
\begin{aligned}
& \varepsilon \simeq 1+\frac{\omega_{p e}^{2}}{\omega_{c}^{2}}-\frac{\omega_{p i}^{2}}{\omega^{2}}-\frac{\omega_{p H}^{2} \omega_{H}^{2}}{\omega^{2}\left(\omega^{2}-\omega_{H}^{2}\right)}, \\
& \eta \simeq-\frac{\omega_{p e}^{2}}{\omega^{2}}, \quad \varepsilon-\eta \simeq \frac{\omega_{p e}^{2}}{\omega^{2}}-\frac{\omega_{p H}^{2} \omega_{H}^{2}}{\omega^{2}\left(\omega^{2}-\omega_{H}^{2}\right)} .
\end{aligned}
$$

For the frequency range above the largest cutoff frequency $\omega_{\text {cut }}$ (see also Appendix B), we will mainly use an expression of $\digamma(\omega)$ which has a form similar to the simplest one (Eq. 19) (it is clear that in the estimation of the cutoff frequency one should keep not only the contribution of hydrogen ions). Then, in the extra-low whistler frequency domain, the dispersion law $\omega(k, \theta)$ satisfies

$$
\omega^{2}-\omega_{m d}^{2}+\omega^{2} \frac{\tilde{\varkappa}\left(\varkappa+\sin ^{2} \theta\right)}{p} \Delta \digamma-\Omega_{c}^{2} \frac{\widehat{q}}{p} \frac{n_{H}}{n_{e}} \frac{m}{M} \frac{\omega_{H}^{2}}{\omega^{2}-\omega_{H}^{2}}=0,
$$

where $\Delta \digamma=\mu \digamma-1 ; \tilde{\varkappa}$ and $\Omega_{c}^{2}$ are defined in Eq. (26). In more general cases (as two positive ions and negative ion or dust grain, for example) to reduce Eq. (23) finally to a biquadratic equation for $\omega$, the structure of $\Delta \digamma$ should not be more complex than

$$
\Delta \digamma=\frac{\omega^{2}\left(\omega_{H}^{2}-\widehat{\omega}_{\mathrm{cut} 1}^{2}-\widehat{\omega}_{\mathrm{cut} 2}^{2}\right)+\widehat{\omega}_{\mathrm{cut} 1}^{2} \widehat{\omega}_{\mathrm{cut} 2}^{2}}{\omega^{2}\left(\omega^{2}-\omega_{H}^{2}\right)} .
$$

In the above expression, however, one should provide the data about the two highest ion cutoff frequencies $\widehat{\omega}_{\text {cut } 1}^{2}>\widehat{\omega}_{\text {cut } 2}^{2}$, in comparison with Eq. (19), which is applicable if $\omega^{2} \gtrsim \omega_{\text {cut }}^{2}=\widehat{\omega}_{\text {cut } 1}^{2} \gg \widehat{\omega}_{\text {cut } 2}^{2}$ (see also Appendix B). One can compare $\widehat{q}=q-\cos ^{2} \theta$ in Eq. (23) with the parameter $q$ appearing in the expression of the modified electron whistler dispersion law (Lundin and Krafft, 2002)

$$
\omega_{m d}^{2}=\frac{\Omega_{c}^{2}}{p}\left(\cos ^{2} \theta+q \delta_{i}\right), \quad \delta_{i}=\frac{\omega_{p i}^{2}}{\omega_{p e}^{2}}=\frac{\omega_{l h r}^{2}}{\Omega_{c}^{2}},
$$

where

$$
\begin{aligned}
p= & (1+\varkappa)(1+\tilde{\varkappa})+\mu \tilde{\varkappa} \cos ^{2} \theta, \\
& \tilde{\varkappa}=\varkappa /(1+\mu), \\
q= & 1+\varkappa+\varkappa \cos ^{2} \theta, \quad \mu=\omega_{c}^{2} / \omega_{p e}^{2}, \\
& \Omega_{c}^{2}=\omega_{c}^{2} /(1+\mu),
\end{aligned}
$$

and

$$
\begin{aligned}
\omega_{l h r}^{2} & \equiv \frac{\mu}{1+\mu} \omega_{p i}^{2}=\omega_{H} \omega_{c} \frac{\omega_{p}^{2}}{\omega_{c}^{2}+\omega_{p e}^{2}}(Z / M)_{\mathrm{eff}}, \\
\omega_{p i}^{2} & \equiv \sum_{j} \omega_{p j}^{2},
\end{aligned}
$$

with an effective specific charge of the ion population $(Z / M)_{\text {eff }}$ defined by Eq. (8).
Let us estimate the typical values of the wavelengths, or $\varkappa=\omega_{p e}^{2} / k^{2} c^{2}$, corresponding to the different frequency domains which are spanned by the largest root of Eq. (23). The high frequency limit of Eq. (23), when $\digamma \simeq 1 / \mu$ (Eq. 9) and $\varepsilon \simeq 1+1 / \mu-\omega_{p i}^{2} / \omega^{2}$, leads to the modified whistler dispersion law (Eq. 25), $\omega^{2} \simeq \omega_{m d}^{2} \gg \omega_{H}^{2}$. This frequency domain near or above $\omega_{l h r}$ can be covered for values $\varkappa \lesssim \sqrt{M / m}$, so that $q / p$ (Eq. 26) belongs to the interval $\sqrt{m / M} \lesssim q / p \lesssim 1$. However, to approach the extra-low-frequencies close to $\omega_{H}$ when the wave propagation is oblique with respect to $\boldsymbol{B}_{0}$, one should provide rather large $\varkappa$, i.e. $\varkappa \simeq M / m \gg 1$ (or $\omega_{p H}^{2} / k^{2} c^{2} \simeq 1$ ) so that $p \simeq \varkappa^{2} /(1+\mu) \gg q \simeq \varkappa \gg 1$ and $q / p \sim m / M$; then the value of $\omega_{m d}$ (Eq. 25) contributing to (23) can reach $\omega_{H}$

$\omega_{m d}^{2} \simeq \frac{\Omega_{c}^{2}}{p} \simeq \omega_{H}^{2}>\frac{q}{p} \omega_{l h r}^{2}=\frac{q}{p} \frac{\omega_{H} \omega_{c}}{1+\mu} M_{\mathrm{eff}}^{-1} \simeq \frac{\omega_{H}^{2}}{1+\mu} M_{\mathrm{eff}}^{-1}$.

Substituting the simplest form of $\Delta \digamma$ (corresponding to Eq. 19) into Eq. (23), one obtains the biquadratic equation

$$
\begin{gathered}
\omega^{4}-\omega^{2}\left[\omega_{m d}^{2}+\omega_{H}^{2}-\frac{\tilde{\varkappa}\left(\varkappa+\sin ^{2} \theta\right)}{p}\left(\omega_{H}^{2}-\omega_{\mathrm{cut}}^{2}\right)\right] \\
+\omega_{H}^{2}\left(\omega_{m d}^{2}-\Omega_{c}^{2} \frac{\widehat{q}}{p} \frac{n_{H}}{n_{e}} \frac{m}{M}\right)=0 .
\end{gathered}
$$

Then the largest root of Eq. (29), where $\varkappa$ stretches over the interval $1 \lesssim \varkappa \lesssim M / m$, spans over the full electron whistler frequency domain, while the smallest root describes the branch with a frequency lower than $\omega_{H}$ and a resonance near $\omega_{H}$.

\section{Numerical fitting and conclusions}

Let us now demonstrate by numerical calculations that the biquadratic dispersion equation presented in Eqs. (23-24) is relevant, due to its very good agreement with the electron whistler mode branch dispersion law in a cold plasma, at any angle of propagation. Actually, the most visible difference arises mainly in the high frequency domain when the ratio $\omega / \omega_{p e}$ becomes finite and not small; however, in this case some noticeable difference between the total cold plasma and the new or the modified dispersion laws, is expectable (see Eqs. 9, 10).

For definiteness, let us present the example of a plasma with finite $\omega_{p e} / \omega_{c}$, corresponding to the conditions of the upper ionosphere in the transition region from the oxygen to the hydrogen ions' prevalence. The spatial plasma density distributions are derived from a diffusive equilibrium density model (Angerami and Thomas, 1964; Kimura 1985) with two ions $\left(\mathrm{O}^{+}\right.$and $\left.\mathrm{H}^{+}\right)$. The altitude $h_{0}$ where both ions' densities are equal, $n_{H}=n_{O}$ (or $v_{H} \equiv n_{H} / n=v_{O}=0.5$ ), is selected as $h_{0}=1800 \mathrm{~km}$; the 


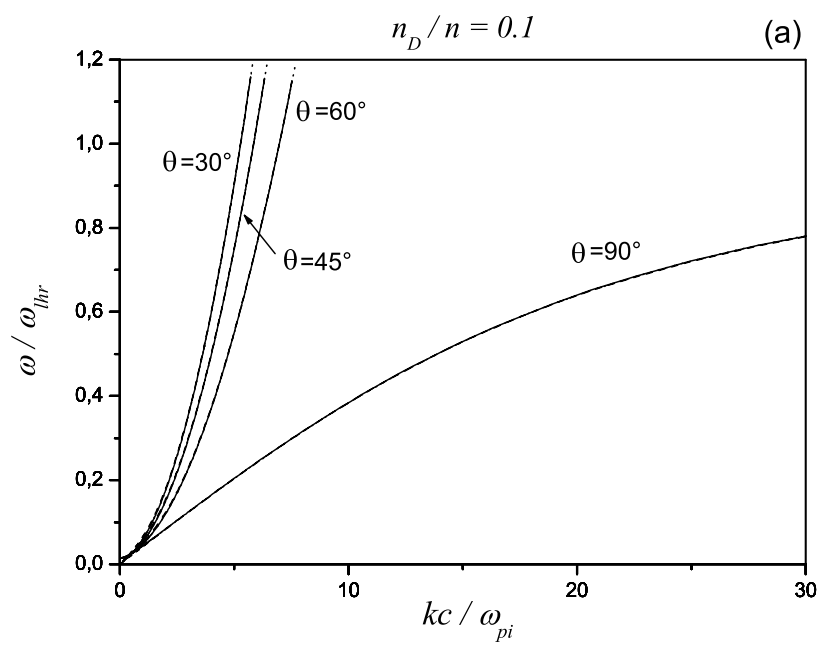

Fig. 1a. Dispersion laws $\left(\omega / \omega_{l h r}\right.$ as a function of $\left.k c / \omega_{p i}\right)$ for different wave vector orientations with respect to the magnetic field $(\theta=30,45,60$, and 90 degrees $)$ in a plasma with two positive ions $\mathrm{H}^{+}, \mathrm{O}^{+}$, at an altitude $h \simeq 1900 \mathrm{~km}$, and with a negative $\mathrm{O}^{-}$relative content of $n_{D} / n=0.1$. Solid lines: new dispersion law; dotted lines: whistler solution of the total cold plasma dispersion law; dashed lines: modified whistler dispersion law $\omega_{m d}$. Main parameters are: $\omega_{\text {cut }} \simeq \widehat{\omega}_{\text {cut } 1} \simeq 0.29 \omega_{H}, \widehat{\omega}_{\text {cut } 2} \simeq 0.1 \omega_{H}, \omega_{H} / 2 \pi \simeq 0.21 \mathrm{kHz}$.

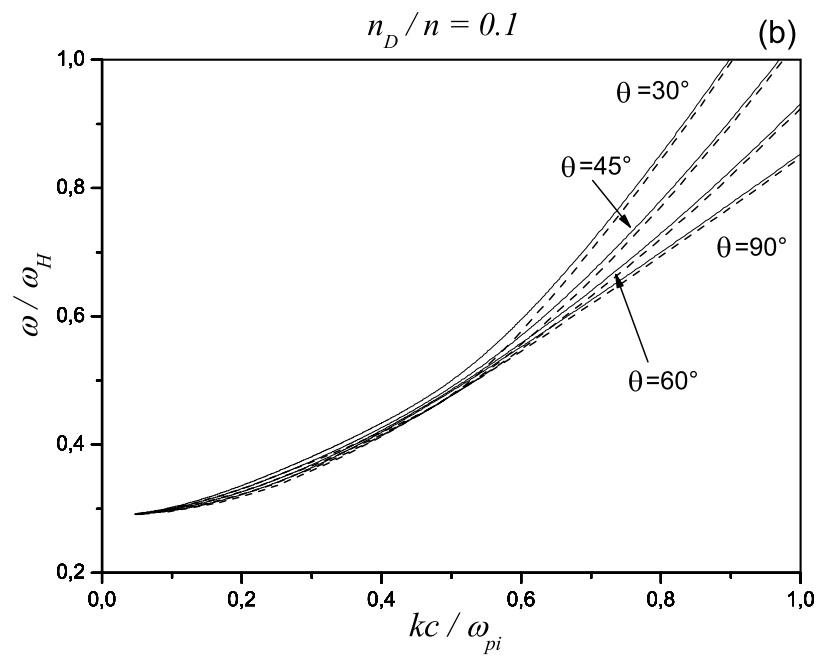

Fig. 1b. Total cold and new dispersion laws (solid and dotted lines, respectively), for the parameters of Fig. 1a but with another frequency scale: $\omega / \omega_{H}$ as a function of $k c / \omega_{p i}$.

value of the electron plasma frequency is selected at the base level of $h=1000 \mathrm{~km}$ as $\omega_{p e} / 2 \pi \simeq 963 \mathrm{kHz}$ (corresponding to $n_{e}=1.15 \times 10^{10} \mathrm{~cm}^{-3}$ ); the characteristic scale of the altitude distribution is governed by the electron $T_{e}$ and the ion temperatures, $T_{i}=T_{e} / 2 \simeq 0.1 \mathrm{eV}$ (more details can be found in Lundin and Krafft, 2001). The plasma parameters at $h_{0}=1800 \mathrm{~km}$ are presented in Table 1 (with

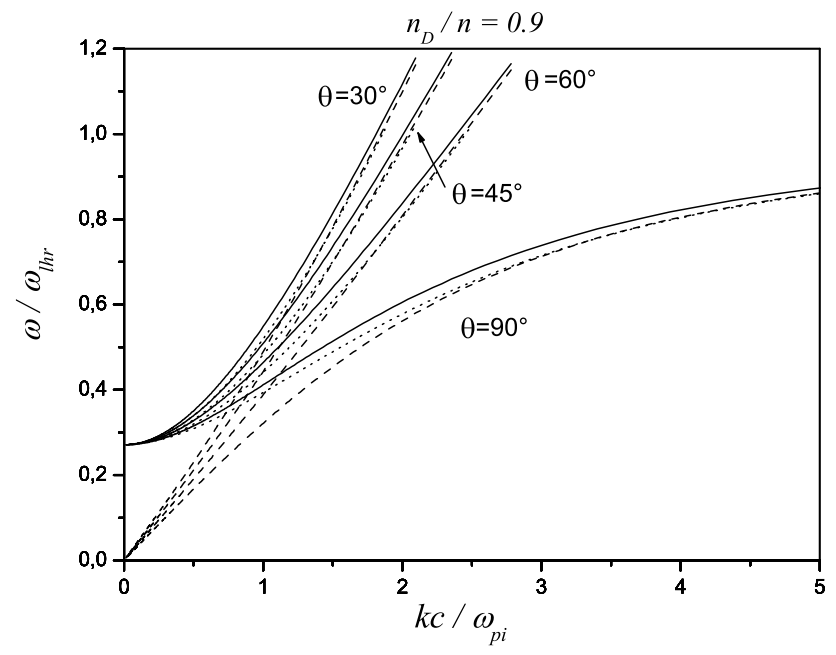

Fig. 2. Dispersion laws $\left(\omega / \omega_{l h r}\right.$ as a function of $\left.k c / \omega_{p i}\right)$ for different wave vector orientations with respect to the magnetic field $(\theta=30,45,60$, and 90 degrees) in a plasma with two positive ions $\mathrm{H}^{+}, \mathrm{O}^{+}$, at an altitude $h \simeq 1900 \mathrm{~km}$, and with a negative $\mathrm{O}^{-}$relative content of $n_{D} / n=0.9$. Main parameters are: $\omega_{\text {cut }} \simeq \widehat{\omega}_{\text {cut } 1} \simeq 6.26 \omega_{H}, \widehat{\omega}_{\text {cut } 2} \simeq 0.11 \omega_{H}, \omega_{H} / 2 \pi \simeq 0.21 \mathrm{kHz}$.

$\left.\omega_{p e}=\omega_{p}, \omega_{H} \simeq 0.22 \mathrm{kHz}, \omega_{\text {cut }} \simeq 0.53 \omega_{H}\right)$. Then for the near equatorial region to a fixed magnetic latitude $\lambda=5^{\circ}$, the plasma parameters for different altitudes $h$ are shown in Table 1. At $h=2500 \mathrm{~km}$ (resp. $h=1400 \mathrm{~km}$ ), the plasma consists of almost purely hydrogen (resp. oxygen) ions. The LHR frequency maximum, $\omega_{l h r} / 2 \pi \simeq 4.36 \mathrm{kHz}$, is realized at the altitude $h=2100 \mathrm{~km}$, where $v_{H} \simeq 0.93, v_{O} \simeq 0.07$, $\omega_{p e} / 2 \pi=228 \mathrm{kHz}, \omega_{c}^{2} / \omega_{p e}^{2}=2.56, \omega_{H} / 2 \pi \simeq 0.2 \mathrm{kHz}$, and $\omega_{\text {cut }} \simeq 0.126 \omega_{H}$. The plasma parameters corresponding to $h=1900 \mathrm{~km}$ are also presented in Table 1 $\left(\omega_{H} / 2 \pi \simeq 0.21 \mathrm{kHz}, \omega_{\text {cut }} \simeq \widehat{\omega}_{\text {cut } 1} \simeq 0.33 \omega_{H}\right)$; this altitude is above the altitude of equipartition between $\mathrm{H}^{+}$and $\mathrm{O}^{+}$but lower than that corresponding to the LHR frequency maximum.

To examine the influence of the negative ions' density on the electron whistler dispersion law, let us use the same background plasma parameters (i.e. the relative densities of all the positive ions) at the altitude $h=1900 \mathrm{~km}$, except for the relative electron density $v_{e}$, which is reduced by selecting an appropriate relative content $v_{D}$ of negative heavy particles - for definiteness, let it be $\mathrm{O}^{-}$ions. Table 2 corresponds to plasma parameters for two values of the relative negative ions amount, $v_{D}=0.9$ and $v_{D}=0.1$.

Figures $1 \mathrm{a}$ and $\mathrm{b}\left(\omega / \omega_{l h r}\right.$ and $\omega / \omega_{H}$ as a function of $k c / \omega_{p i}$, respectively) allow one to compare the three dispersion laws: the new dispersion law (solid lines), the total cold plasma dispersion law (dotted lines in Figs. 1a and 2; dashed lines in Fig. 1b) and the so-called modified dispersion law (dashed lines), for $n_{D} / n=0.1$ and four different angles of the wave vector, namely $\theta=30,45,60$ and 90 degrees 
Table 1. $v_{H}$ and $v_{O}$ are the relative content of Hydrogen and Oxygen, respectively; $\omega_{p e} / 2 \pi$ and $\omega_{l h r} / 2 \pi$ are the electron plasma and the LHR frequencies in $\mathrm{kHz} ; \omega_{c}^{2} / \omega_{p e}^{2}$ is the square of the electron gyrofrequency to the electron plasma frequency of the background plasma; $v_{D}=0$ corresponds to the absence of negative ions. These values are presented for the set of altitudes indicated in the first column (in km) and for a fixed latitude of 5 degrees.

\begin{tabular}{ccccccc}
\hline$h(\mathrm{~km})$ & $v_{H}$ & $v_{O}$ & $\omega_{p e} / 2 \pi(\mathrm{kHz})$ & $\omega_{l h r} / 2 \pi(\mathrm{kHz})$ & $\omega_{c}^{2} / \omega_{p e}^{2}$ & $v_{D}$ \\
\hline 2500 & 0.997 & 0.003 & 217 & 4.2 & 2.13 & 0 \\
1900 & 0.71 & 0.29 & 243 & 4.11 & 2.6 & 0 \\
1800 & 0.5 & 0.5 & 260.2 & 3.73 & 2.44 & 0 \\
1400 & 0.02 & 0.98 & 457 & 2.2 & 1.1 & 0 \\
\hline
\end{tabular}

Table 2. The parameters of Table 1 are presented at the altitude of $1900 \mathrm{~km}$ for two values of the relative content $v_{D}$ of the negative ions $\mathrm{O}^{-}$ (last column).

\begin{tabular}{ccccccc}
\hline$h(\mathrm{~km})$ & $v_{H}$ & $v_{O}$ & $\omega_{p e} / 2 \pi(\mathrm{kHz})$ & $\omega_{l h r} / 2 \pi(\mathrm{kHz})$ & $\omega_{c}^{2} / \omega_{p e}^{2}$ & $v_{D}$ \\
\hline 1900 & 0.71 & 0.29 & 76.9 & 4.93 & 26 & 0.9 \\
1900 & 0.71 & 0.29 & 230.6 & 4.19 & 2.9 & 0.1 \\
\hline
\end{tabular}

$\left(\omega_{\text {cut }} \simeq \widehat{\omega}_{\text {cut } 1} \simeq 0.29 \omega_{H}, \widehat{\omega}_{\text {cut } 2} \simeq 0.1 \omega_{H}\right)$. Figure 2 shows the three dispersion laws by presenting $\omega / \omega_{l h r}$ as a function of $k c / \omega_{p i}$, for $n_{D} / n=0.9$ and the same wave vector angles $\theta$ as in Fig. $1\left(\omega_{\text {cut }} \simeq \widehat{\omega}_{\text {cut } 1} \simeq 6.26 \omega_{H}, \widehat{\omega}_{\text {cut } 2} \simeq 0.11 \omega_{H}\right)$.

Moreover, Fig. 3 represents the 2-D profile of the ratio of the highest ion cutoff frequency to the LHR frequency as a function of the relative negative ion charge content $Z_{D} n_{D} / n$ and the inverse specific charge of the negative ion $M_{D}($ a.u. $) / Z_{D}=\left(M_{D} / M\right) / Z_{D}$ for the plasma parameters corresponding to the altitude $h=1900 \mathrm{~km}$ (represented only for $M_{D}($ a.u. $) / Z_{D}>4$, where the error of simple analytical estimates of $\omega_{\text {cut }}$ (Eqs. B4, B9) is small enough).

In all the figures one can see that even the modified electron whistler dispersion law $\omega_{m d}$ (dashed lines) provides a rather good approximation of the total cold dispersion law, in the frequency domain extending till the ions' gyrofrequency: the value of the frequency splitting between the curves does not exceed the value of the proton gyrofrequency; however, it cannot reproduce the details of the dispersion curve near the cutoff frequency.

Thus, under rather general conditions, we have found an expression for the low frequency whistler dispersion law which requires the value of the electron gyrofrequency, the electron plasma frequency, the LHR frequency, the highest ion cutoff frequency and the relative content of the lightest ion only. Except for the relative lightest ion content, all other background plasma parameters can be found in the spectral peculiarities of the electron whistler electromagnetic emissions registered on board the satellites. The more complex case when several light ions of comparable gyrofrequencies and the heavy ions' background contribute together to the whistler dispersion can also be investigated by the above ap-

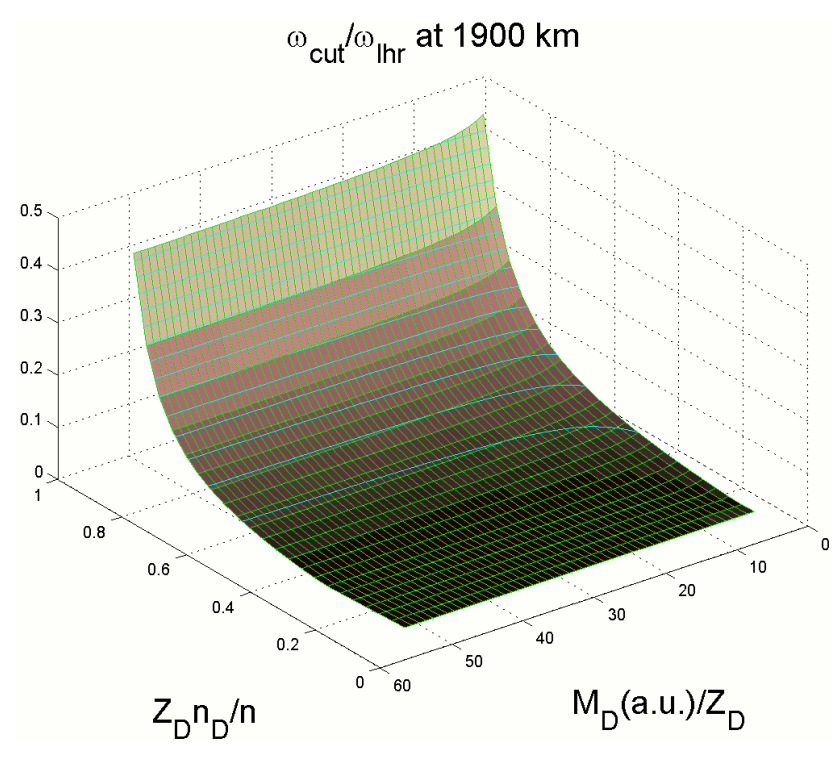

Fig. 3. The 2-D profile of the ratio of the highest ion cutoff frequency to the LHR frequency as a function of the relative negative ion charge content $Z_{D} n_{D} / n$ and the inverse specific charge of negative ion $M_{D}($ a.u. $) / Z_{D}=\left(M_{D} / M\right) / Z_{D}$ (in the interval $M_{D}$ (a.u.) $/ Z_{D}>4$ ) for plasma parameters corresponding to the altitude $h \simeq 1900 \mathrm{~km}$.

proach. However, after relevant simplification one should keep in $\digamma$ the resonant terms for all the light ions; then the dispersion equation should be solved conventionally as a biquadratic equation for the refractive indexes.

We should stress that our dispersion law is well applicable also in the case when the content of negative ions or negative 
dust grains is large, when the highest ion cutoff frequency governing the electron whistler dispersion law essentially exceeds the lightest ion gyrofrequency and can be rather close to the LHR frequency $\omega_{l h r}$ (around $\sim 0.3 \omega_{l h r}$, in comparison with the conventional value around $\sim 0.02 \omega_{l h r}$, for example). This high value of the cutoff frequency will result in the reflection of rather high frequency whistler waves at low altitudes. At the same time, an essential variation of the electron density and the LHR frequency in the local regions enriched by negative ions of electronegative gases (even as a transient state) also can lead to the observable features of the registered frequency-time electron whistler spectra in the ELF-VLF domain. The propagation of whistler wave packets through the space regions with a noticeable amount of negative ions (even if it is a transient state of localized regions) can be investigated by usual ray-tracing codes; the features of possibly registered sonograms will be studied in forthcoming papers.

\section{Appendix A}

\section{Modified electron whistler dispersion law}

Let us remember that the usual dispersion equation for waves propagating in a cold plasma (without any approximation) can be written in the following form (Lundin and Krafft, 2001, 2002)

$\cos ^{2} \theta\left(1-\frac{\chi}{N^{2}}\right)=\left(1-\frac{\eta}{N^{2}}\right)\left(\cos ^{2} \theta_{R}\left(1-\frac{\eta}{N^{2}}\right)-\frac{\chi}{N^{2}}\right)$,

where $N$ is the refractive index and $\theta$ is the angle defined by $\cos \theta \equiv \boldsymbol{k} \cdot \boldsymbol{B}_{0} / k B_{0} ; \chi, \eta$ and $\cos ^{2} \theta_{R}$ are defined in the text by Eqs. (2-4). In the high frequency limit, when the contribution of the ions to the dielectric permittivity tensor is negligible, one has $(\varepsilon-1)(\varepsilon-\eta)=g^{2}$ (Wieder, 1964) and

$\chi=1, \quad \cos ^{2} \theta_{R}=\frac{\omega^{2}(1+\mu)}{\omega_{c}^{2}}\left(1-\frac{\omega^{2}}{\omega_{p e}^{2}(1+\mu)}\right)$,

$\mu=\frac{\omega_{c}^{2}}{\omega_{p e}^{2}}$,

where the tensor components $\varepsilon$ and $g$ as well as the frequencies $\omega_{p e}$ and $\omega_{c}$ are defined in the text by Eqs. (4-5). Then, the dispersion law of the electron whistler waves for a finite value of $\mu$ and for frequencies $\omega \ll \omega_{p e}$ can be written in the form

$\omega^{2}=\frac{\Omega_{c}^{2} \cos ^{2} \theta}{(1+\varkappa)(1+\tilde{\varkappa})+\mu \tilde{\varkappa} \cos ^{2} \theta}$,

with $\cos ^{2} \theta_{R} \simeq \omega^{2} / \Omega_{c}^{2}$ and

$$
\begin{aligned}
\Omega_{c}^{2} & =\omega_{c}^{2} /(1+\mu), \quad \Omega_{p e}^{2}=\omega_{p e}^{2} /(1+\mu), \\
\varkappa & =\omega_{p e}^{2} / k^{2} c^{2}, \quad \tilde{\varkappa}=\Omega_{p e}^{2} / k^{2} c^{2} .
\end{aligned}
$$

In an overdense plasma where $\mu \ll 1$, the conventional whistler dispersion law can be recovered from Eq. (A3), namely

$\omega^{2}=\frac{\omega_{c}^{2} \cos ^{2} \theta}{(1+\varkappa)^{2}}$.

In the frequency domain near $\omega_{l h r}\left(\omega_{H} \ll \omega \ll \omega_{c}\right)$, the parameters of Eq. (10) correspond to the following expressions

$$
\begin{aligned}
\chi & =1-\frac{\omega_{p i}^{2}}{\omega^{2}}, \quad \cos ^{2} \theta_{R}=\frac{\omega^{2}}{\Omega_{c}^{2}}\left(1-\frac{\omega_{l h r}^{2}}{\omega^{2}}\right), \\
\varepsilon & \simeq 1+\frac{\omega_{p e}^{2}}{\omega_{c}^{2}}-\frac{\omega_{p i}^{2}}{\omega^{2}}=\left(1+\frac{1}{\mu}\right)\left(1-\frac{\omega_{l h r}^{2}}{\omega^{2}}\right), \\
\omega_{l h r}^{2} & =\frac{\mu}{(1+\mu)} \omega_{p i}^{2}, \quad \omega_{p i}^{2}=\sum_{j} \omega_{p j}^{2}, \quad \mu=\frac{\omega_{c}^{2}}{\omega_{p e}^{2}},
\end{aligned}
$$

so that the modified electron whistler dispersion law (Eq. 25) can be recovered from Eq. (A1).

\section{Appendix B}

\section{Plasma containing three ion species of different charge signs}

Let us consider a rather general case which includes most features of the considered phenomena, that is, a plasma containing negative ions (dust particles, labeled by ' $D$ ') and two positive ions' species (hydrogen and oxygen ions, for example, labeled ' 1 ' and ' 2 ', respectively). Owing to Eq. (12) one obtains

$$
\varepsilon \pm g=1-\omega_{p e}^{2} \sum_{i=1}^{4} \frac{v_{i} \omega_{i}}{v_{e} \omega_{c}} \frac{\left(\omega \pm \omega_{\mathrm{cut} 1}\right)\left(\omega \pm \omega_{\mathrm{cut} 2}\right)}{\left(\omega \pm \omega_{c}\right)\left(\omega \pm \omega_{D}\right)\left(\omega \mp \omega_{1}\right)\left(\omega \mp \omega_{2}\right)},
$$

where the summation over $i$ includes electrons, as well as positive and negative ions; the relative particles' densities

$v_{i}=\frac{Z_{i} n_{i}}{n}$,

verify the charge neutrality condition

$$
1=\sum_{\alpha} v_{\alpha}=v_{e}+\sum_{\beta} v_{\beta}
$$

The frequencies $\omega_{\text {cut } 1}$ and $\omega_{\text {cut } 2}$ are the roots of the quadratic equation corresponding to $\varepsilon-g \simeq \varepsilon-g-1=0$, that is

$$
\begin{aligned}
& \omega^{2} \sum_{i=1}^{4} v_{i} \omega_{i}-\omega \omega_{c}\left[v_{D} \omega_{D}+v_{2} \omega_{2}+v_{1} \omega_{1}-v_{e}\left(\omega_{1}+\omega_{2}-\omega_{D}\right)\right] \\
& -\omega\left[-\left(v_{1}+v_{2}\right) \omega_{1} \omega_{2}-\left(v_{2}-v_{e}\right) \omega_{1} \omega_{D}-\left(v_{1}-v_{e}\right) \omega_{2} \omega_{D}\right]
\end{aligned}
$$


$-v_{e} \omega_{1} \omega_{2} \omega_{D}-\omega_{c}\left[v_{1} \omega_{2} \omega_{D}+v_{2} \omega_{1} \omega_{D}+v_{D} \omega_{1} \omega_{2}\right]=0$

whereas $-\omega_{\text {cut1 }}$ and $-\omega_{\text {cut2 }}$ are the roots of $\varepsilon+g \simeq \varepsilon+g-1=0$; in accordance with Eq. (6), one can use the relation

$$
\sum_{i=1}^{4} v_{i} \omega_{i}=v_{e} \omega_{c}\left(1+\sum_{j=1}^{3} \frac{\omega_{p j}^{2}}{\omega_{p e}^{2}}\right)=v_{e} \omega_{c}\left(1+\frac{\omega_{p i}^{2}}{\omega_{p e}^{2}}\right) \simeq v_{e} \omega_{c} .
$$

$\omega_{\text {cut } 1}$ and $\omega_{\text {cut } 2}$ are real and verify $\omega_{\text {cut } 1} \omega_{\text {cut } 2}<0$; the largest root $\omega_{\text {cut1 }}$ (for definiteness) of the equation $\varepsilon-g \simeq 0$ is positive while $\omega_{\text {cut } 2}$ is negative, but $-\omega_{\text {cut } 2}>0$ is a root of $\varepsilon+g \simeq 0$. Thus, in the presence of three different ions one has two positive low frequencies, $\omega_{\text {cut } 1}$ and $-\omega_{\text {cut } 2}$, which correspond to two different positive ion cutoff frequencies for which $\varepsilon^{2}-g^{2} \simeq 0$. Actually, the roots $\omega_{\text {cut } 1}$ and $-\omega_{\text {cut2 } 2}$ fit the equation $\varepsilon^{2}-g^{2} \simeq 0$ with a rather reliable accuracy, except for the case of a plasma with a large amount of heavy negatively charged particles, where $v_{e}=n_{e} / n \ll 1$ (see also Eq. 7).

In this case, the value of the upper ion cutoff frequency $\omega_{\text {cut }}$ differs from $\omega_{\text {cut } 1}$ (which is the biggest root of Eq. B4) and can essentially exceed $\omega_{H}$ and even approach $\omega_{l h r}$, so that a more accurate analytical estimate for $\omega_{\text {cut }}$ should be used (if the highest ion cutoff frequency $\omega_{\text {cut }} \gg\left|\omega_{\text {cut } 2}\right|$ is not available from the experiment).

Let us find this correction to the value of $\omega_{\text {cut } 1}$, accompanied by the necessary modification of $\digamma$ (as well as $\Delta \digamma$ ), in a plasma with $n_{e} / n \ll 1$, due to some large amount of electronegative heavy particles. Using that $\omega \ll \omega_{c}$ and Eq. (B5), one can find from Eq. (B1)

$$
\begin{aligned}
-\eta \digamma & =-\left(\varepsilon^{2}-g^{2}\right) \\
& \simeq\left(\omega_{c} \frac{\omega_{p e}^{2}}{\omega_{c}^{2}}\right)^{2} \frac{\left(\omega^{2}-\omega_{\text {cut } 1}^{2}\right)\left(\omega^{2}-\omega_{\text {cut } 2}^{2}\right)}{\left(\omega^{2}-\omega_{D}^{2}\right)\left(\omega^{2}-\omega_{1}^{2}\right)\left(\omega^{2}-\omega_{2}^{2}\right)} \\
& -\omega_{c} \frac{\omega_{p e}^{2}}{\omega_{c}^{2}} \frac{2 \omega^{2}\left[-\omega^{2}\left(\omega_{\text {cut } 1}+\omega_{\text {cut } 2}+\omega_{1}\right)-\omega_{1} \omega_{\text {cut } 1} \omega_{\text {cut } 2}\right]}{\left(\omega^{2}-\omega_{D}^{2}\right)\left(\omega^{2}-\omega_{1}^{2}\right)\left(\omega^{2}-\omega_{2}^{2}\right)} \\
& -1,
\end{aligned}
$$

where, in the numerator of the second term (which is important only for small $n_{e} / n$ and thus for large $\left.\omega_{\text {cut } 1}\right)$, we took into account the condition $\omega \gtrsim \omega_{\text {cut } 1} \gg \omega_{1} \gg \omega_{D}, \omega_{2}$.

To derive a biquadratic dispersion equation similar to Eq. (23), let us use the correction $\Delta \digamma \equiv \digamma \omega_{c}^{2} / \omega_{p e}^{2}-1$ (in the high frequency approximation we have $\digamma \simeq \omega_{p e}^{2} / \omega_{c}^{2}$ ); then, applying Eqs. (21-22), one obtains from Eq. (20) that

$$
\begin{gathered}
\omega^{2}-\omega_{m d}^{2}+\tilde{\varkappa}\left(\varkappa+\sin ^{2} \theta\right) \frac{\omega^{2}}{p} \Delta \digamma-\Omega_{c}^{2} \frac{\omega_{p H}^{2}}{\omega_{p e}^{2}} \frac{\omega_{H}^{2}}{\left(\omega^{2}-\omega_{H}^{2}\right)} \\
\times \frac{\left(q-\cos ^{2} \theta\right)}{p}=0,
\end{gathered}
$$

$$
\begin{aligned}
\Delta \digamma \simeq & -\frac{\omega^{2}}{\omega_{p e}^{2}} \mu-1+\frac{\left(\omega^{2}-\omega_{\mathrm{cut} 1}^{2}\right)\left(\omega^{2}-\omega_{\mathrm{cut} 2}^{2}\right)}{\omega^{2}\left(\omega^{2}-\omega_{H}^{2}\right)} \\
& +\frac{2 \omega_{c}\left[\omega^{2}\left(\omega_{\mathrm{cut} 1}+\omega_{\mathrm{cut} 2}+\omega_{H}\right)+\omega_{H} \omega_{\mathrm{cut} 1} \omega_{\mathrm{cut} 2}\right]}{\omega_{p e}^{2}\left(\omega^{2}-\omega_{H}^{2}\right)},
\end{aligned}
$$

where we finally apply the condition $\omega^{2} \gg \omega_{D}^{2}, \omega_{2}^{2}$ to all the denominator terms and put $\omega_{1}=\omega_{H}$.

If the function $\Delta \digamma$ can be presented in the form

$\Delta \digamma \propto \frac{a \omega^{4}+b \omega^{2}+c}{\omega^{2}\left(\omega^{2}-\omega_{1}^{2}\right)}$,

where $a, b, c$ and $\omega_{1}^{2}$ are constants, then the resultant dispersion law (Eq. B6) has the form of a biquadratic equation for $\omega$, as Eq. (23). To provide the form (B8), it is sufficient to omit the first term in Eq. (B7), i.e. $\mu \omega^{2} / \omega_{p e}^{2}$. This can be done if the characteristic cutoff frequencies inherent to the ion gyrofrequency domain are sufficiently distant from $\omega_{l h r}$, i.e. if $\max \left\{\omega_{1}, \omega_{\text {cut } 1},\left|\omega_{\text {cut } 2}\right|\right\} \ll \omega_{\text {lhr }}$, which is not valid only when $\omega_{\text {cut } 1}$ is very large, in a plasma with a small amount of free electrons $n / n_{e} \gtrsim \omega_{p} / \omega_{p i} \gtrsim 50$ (see Eqs. B15-B16). In this case we have

$$
\begin{aligned}
\Delta \digamma & \simeq \frac{\omega^{2}\left(\omega_{1}^{2}-\omega_{\text {cut } 1}^{2}-\omega_{\text {cut } 2}^{2}\right)+\omega_{\text {cut } 1}^{2} \omega_{\text {cut } 2}^{2}}{\omega^{2}\left(\omega^{2}-\omega_{1}^{2}\right)} \\
& +2 \mu \frac{\left[\omega^{2}\left(\omega_{\text {cut } 1}-\left|\omega_{\text {cut } 2}\right|+\omega_{1}\right)-\omega_{1} \omega_{\text {cut } 1}\left|\omega_{\text {cut } 2}\right|\right]}{\omega_{c}\left(\omega^{2}-\omega_{1}^{2}\right)},
\end{aligned}
$$

so that one can use the presentation

$$
\begin{aligned}
\mu \digamma-1 & =\Delta \digamma \simeq \frac{\left(\omega^{2}-\widehat{\omega}_{\mathrm{cut} 1}^{2}\right)\left(\omega^{2}-\widehat{\omega}_{\mathrm{cut} 2}^{2}\right)}{\omega^{2}\left(\omega^{2}-\omega_{1}^{2}\right)}-1 \\
& =\frac{\omega^{2}\left(\omega_{1}^{2}-\widehat{\omega}_{\mathrm{cut} 1}^{2}-\widehat{\omega}_{\mathrm{cut} 2}^{2}\right)+\widehat{\omega}_{\mathrm{cut} 1}^{2} \widehat{\omega}_{\mathrm{cut} 2}^{2}}{\omega^{2}\left(\omega^{2}-\omega_{1}^{2}\right)},
\end{aligned}
$$

where the corrected cutoff frequencies $\widehat{\omega}_{\text {cut } 1}$ and $\widehat{\omega}_{\text {cut2 }}$ can be estimated through $\omega_{\text {cut1 }}$ and $\left|\omega_{\text {cut } 2}\right|$ using Eq. (B9) in the equation $\mu \digamma=\Delta \digamma+1=0$, or can be taken from the experiment; then Eq. (B10) can be used in Eq. (B6).

At last, let us consider the case when the relative electron density is very small

$$
n_{e} / n=v_{e} \ll v_{1}+v_{2}=1 \simeq v_{D}, \quad \omega_{1}>\omega_{2}, \omega_{D},
$$

in the presence of extra electronegative dust grains which collect most part of the negative charge, i.e. $v_{D} \simeq 1$, when the cutoff frequency $\widehat{\omega}_{\text {cut1 }}$ (and $\omega_{\text {cut1 } 1}$ ) can even tend to $\omega_{l h r} \simeq \omega_{p i} \quad\left(\omega_{p e}^{2} / \omega_{c}^{2} \sim n_{e} / n \ll 1\right)$. To estimate $\omega_{\text {cut1 }}$ using $\varepsilon-g \simeq \varepsilon-g-1=0$ (B1), let us take into account that it is not very close to $\left|\omega_{\text {cut } 2}\right|$, so that formally the roots of $a x^{2}+b x+c=0$ (Eq. B4) under the condition $4 a c \ll b^{2}$ can be 
found as $x_{1} \simeq-b / a$ and $x_{2} \simeq-c / b$, that is

$$
\begin{aligned}
\omega_{\mathrm{cut} 1} & \simeq\left(\frac{\nu_{1}}{v_{e}}-1\right) \omega_{1}+\left(\frac{\nu_{2}}{v_{e}}-1\right) \omega_{2}+\frac{n}{n_{e}} \omega_{D} \\
& \simeq \frac{\nu_{1} \omega_{1}+v_{2} \omega_{2}+v_{D} \omega_{D}}{v_{e}}=\frac{n}{n_{e}} \frac{\omega_{c}^{2}}{\omega_{p}^{2}} \frac{\omega_{p i}^{2}}{\omega_{c}}, \\
-\omega_{\text {cut } 2} & \simeq \frac{\omega_{p}^{2}}{\omega_{c}^{2}} \frac{\omega_{c}}{\omega_{p i}^{2}}\left[\left(v_{1} \omega_{2}+v_{2} \omega_{1}\right) \omega_{D}+\omega_{1} \omega_{2}\right] .
\end{aligned}
$$

Then, the condition for sufficiently separated cutoff frequencies, $\omega_{\text {cut } 1} \gg\left|\omega_{\text {cut } 2}\right|$, can be, using Eq. (8), reduced to

$$
\frac{n}{n_{e}} \frac{\omega_{H}}{\omega_{O}}\left[(Z / M)_{\mathrm{eff}}\right]^{2} \gg\left(1+\frac{\omega_{D}}{\omega_{O}}\right),
$$

where $\omega_{O}$ is the gyrofrequency of the oxygen ions.

To validate the presentation of Eqs. (B6) and (B10), let us find the condition for omitting the first term of $\Delta \digamma$ in Eq. (B7). For $\omega^{2} \gtrsim \omega_{\text {cut } 1}^{2} \gg \omega_{1}^{2}, \omega_{\text {cut } 2}^{2}$, and using $\left(\omega_{c}^{2} / \omega_{p e}^{2}\right)\left(n_{e} / n\right)=\omega_{c}^{2} / \omega_{p}^{2} \sim 1$, Eqs. (B7) and (B12) lead to the estimate

$$
\begin{aligned}
\Delta \digamma & \simeq-\frac{\omega^{2}}{\omega_{p e}^{2}} \frac{\omega_{c}^{2}}{\omega_{p e}^{2}}-\frac{\omega_{\text {cut1 }}^{2}}{\omega^{2}}+\frac{2 \omega_{c} \omega_{\text {cut } 1}}{\omega_{p e}^{2}} \\
& \sim \frac{n^{2}}{n_{e}^{2}} \frac{\omega_{p i}^{2}}{\omega_{p}^{2}}\left(-\frac{\omega^{2}}{\omega_{p i}^{2}}-\frac{\omega_{p i}^{2}}{\omega^{2}}+2\right),
\end{aligned}
$$

where the first term inside the brackets corresponds to the first one in Eq. (B7); to neglect it for the frequency interval of interest, one should satisfy $\omega_{\text {cut } 1}^{2} \lesssim \omega^{2} \ll \omega_{l h r}^{2} \simeq \omega_{p i}^{2}$ and thus

$\frac{\omega_{p i}^{2}}{\omega_{\text {cut1 }}^{2}} \simeq \frac{\omega_{p}^{2}}{\omega_{c}^{2}}\left(\frac{n_{e}}{n}\right)^{2} \frac{\omega_{p}^{2}}{\omega_{p i}^{2}} \sim\left(\frac{n_{e}}{n}\right)^{2} \frac{\omega_{p}^{2}}{\omega_{p i}^{2}} \gg 1$,

which leads to

$$
\left(\frac{n_{e}}{n}\right)^{2} \gg \frac{\omega_{p i}^{2}}{\omega_{p}^{2}}=\frac{m}{M}(Z / M)_{\mathrm{eff}} .
$$

Thus, under the condition (B16) there is a rather wide frequency domain $\omega_{\text {cut } 1} \lesssim \omega \ll \omega_{l h r} \lesssim \omega_{p i}$ where the approximation of $\Delta \digamma$, corresponding to the biquadratic Eq. (B6), is valid even for rather small relative electron densities. Figure 3 shows an example of the 2-D contours of the ratio of the highest ion cutoff frequency to the LHR frequency, as a function of the relative negative ion charge content $Z_{D} n_{D} / n$ and the inverse specific charge of negative ions $M_{D}($ a.u. $) / Z_{D}=\left(M_{D} / M\right) / Z_{D}$, in the interval $\left(M_{D} / M\right) / Z_{D}>4$ (see also the text).

Acknowledgements. The authors acknowledge the Centre National de la Recherche Scientifique (CNRS, France) and the Russian Academy of Sciences for their financial support. Useful discussions with B. S. Ryabov are also acknowledged.

Topical Editor I. A. Daglis thanks W. J. Hughes and another anonymous referee for their help in evaluating this paper.

\section{References}

An, T., Merlino, R. L., and D'Angelo, N.: Lower-hybrid waves in a plasma with negative ions, Phys. Fluids B, 5(6), 1917-1918, 1993.

Angerami, J. J. and Thomas, J. O.: Studies of planetary atmospheres, 1. The distribution of electrons and ions in the Earth's exosphere, J. Geophys. Res., 69(21), 4537-4560, 1964.

Benson, R. F., Osherovich, V. A., Fainberg, J., and Reinisch, B. W.: Classification of IMAGE/RPI-stimulated plasma resonances for the accurate determination of magnetospheric electron density and magnetic field values, J. Geophys. Res., 108(A5), 12071220, 2003.

Brice, N. M. and Smith, R. L.: Lower hybrid resonance emissions, J. Geophys. Res., 70(1), 71-80, 1965.

Buchsbaum, S. J.: Resonance in a plasma with two ion species, Phys. Fluids, 3, 418-420, 1960.

Burch, J. L.: IMAGE mission overview, Space Sci. Rev., 91, 1-14, 2000.

Carpenter, D. L.: Remote sensing the Earth's plasmasphere, Radio Sci. B., 308, 19-30, 2004.

Das, G. C. and Uberoi, C.: Crossover frequencies in multicomponent plasmas with negative ions, J. Geophys. Res., 77(28), 55975601, 1972.

Faria Jr., R. T., Mirza, A. M., Shukla, P. K., and Pokhotelov, O. A.: Linear and nonlinear dispersive Alfvén waves in two-ion plasmas, Phys. Plasmas, 5(8), 2947-2951, 1998.

Ganguli, G. and Rudakov, L.: Dynamics in a multicomponent plasma near the low-frequency cutoff, Phys. Rev. Lett., 93(13), $135001,1-4,2004$.

Gintzburg, M. A.: Low-frequency waves in multicomponent plasma, Geomagnetism Aeronomy, 3, 610-614, 1963.

Gurnett, D. A. and Burns, T. B.: The low-frequency cutoff of ELF emissions, J. Geophys. Res., 73, 23, 7437-7445, 1968.

Helliwell, R. A.: Whistlers and Related Ionospheric Phenomena, Stanford University Press, Stanford, Calif., 1965.

Helliwell, R. A.: Forty Years of Whistlers, Modern Radio Science, edited by: Matsumoto, H., Oxford University Press, 189-212, 1993.

Hertzberg, M. P., Cramer, N. F., and Vladimirov, S. V.: Modulational and decay instabilities of Alfven waves in a multicomponent plasma, J. Geophys. Res., 109, A02103, doi:10.1029/2003JA010115, 2004.

Hines, C. O.: Heavy-ion effects in audio-frequency radio propagation, J. Atmos. Terr. Phys., 11, 36-42, 1957.

Irie, S. and Ohsawa, Y.: Quasi-perpendicular magnetosonic waves in a multi-ion-species plasma, Phys. Plasmas, 10(5), 1253-1261, 2003.

Kashyapi, P. K., Chakraborty, B., and Paul, S. N.: Effect of negative ions on nonlinear wave propagation in a magnetized rotating plasma, Phys. Rev. E, 48(4), 2946-2954, 1993.

Kimura, I.: Whistler mode propagation in the Earth and planetary magnetospheres and ray-tracing techniques, Space Sci. Rev., 42, 449-466, 1985.

Lundin, B. V. and Krafft, C.: On the similarity features of normalized frequency spectra of magnetospherically reflected whistlers, J. Geophys. Res., 106(A11), 25 643-25 654, 2001.

Lundin, B. V. and Krafft, C.: Modified electron whistler dispersion law, J. Plasma Phys, 67, parts 2\&3, 149-161, 2002.

Mamun, A. A., Shukla, P. K., and Morfill, G. E.: New cut-off fre- 
quency for low-frequency electromagnetic waves in a multi-ion magnetoplasma with charged dust particulate, Phys. Lett. A, 323, 105-109, 2004.

Mamun, A. A., Shukla, P. K., and Morfill, G. E.: Low-frequency electromagnetic waves in a partially ionized multi-component magnetoplasma, J. Plasma Phys., 71, 389-399, 2005.

Muzzio, J. L. R.: Ion cutoff whistlers, J. Geophys. Res., 73(23), 7526-7529, 1968.

Muzzio, J. L. R. and Angerami, J. J.: Ogo 4 observations of extremely low frequency hiss, J. Geophys. Res., 77(7), 1157-1173, 1972.

Pandey, B. P. and Vranjes, J.: Physics of the dusty Hall plasmas, Phys. Plasmas, 13, 122 106, 1-6, 2006.

Reinish, B. W.: The Radio Plasma Imager investigation on the IMAGE spacecraft, Space Sci. Rev., 91(2), 319-359, 2000.

Santolic, O. and Parrot, M.: Propagation analysis of electromagnetic waves between the helium and proton gyro-frequencies in the low-altitude auroral zone, J. Geophys. Res., 103(A9), 20 469-20 480, 1998.

Sawada, A., Nobata, T., Kishi,Y., Kimura, I., and Oya, H.: Electron density profile in the magnetosphere deduced from in situ electron density and wave normal directions of Omega signals observed by the Akebono (EXOS D) satellite, J. Geophys. Res., 98(7), 11 267-11 274, 1993.

Shafranov, V. D.: Electromagnetic Waves in Plasma, in: Review of Plasma Physics, Consultants Bureau, N.Y., 3, 1-157, 1967.
Shukla, P. K., Kourakis, I., and Stenflo, L.: Low-frequency electromagnetic waves in a Hall-magnetohydrodynamic plasma with charged dust macroparticle, Phys.Plasmas, 12, $024501,1-4$, 2005 .

Smith, J.: Negative ion effects on whistler mode propagation, J. Geophys. Res., 70(1), 53-59, 1965.

Smith, R. L. and Brice, N. M.: Propagation in a multicomponent plasma, J. Geophys. Res., 69(23), 5029-5040, 1964.

Sonwalkar, V. S., Carpenter, D. L., Bell, T. F., et al.: Diagnostics of magnetospheric density and irregularities at altitudes $<5000 \mathrm{~km}$ using whistler and $\mathrm{Z}$ mode echoes from radio sounding on the IMAGE satellite, J. Geophys. Res., 109, A11212, doi:10.1029/2004JA010471, 2004.

Stenzel, R. L.: Whistler waves in space and laboratory plasmas, J. Geophys. Res., 104(A7), 14379-14 395, 1999.

Stix, T. H.: Waves in Plasmas, American Institute of Physics, College Park, Md., 1992.

Wieder, B.: Microwave propagation in an overdense bounded magnetosplasma, Phys. Fluids, 7(7), 964-972, 1964.

Yakimenko, V. L.: Oscillations in a cold plasma containing two ion species, Soviet Phys., Tech. Phys., Engl. Trans., 7(2), 117-124, 1962.

Yu, M. Y., Spatschek, K. H., and Shukla, P. K.: Scattering and modulational instabilities in magnetized plasmas, Z. Naturforsch., A, 29(12), 1736-1741, 1974. 